valid for $|\delta|<1$, or, finally,

$$
\Lambda(\epsilon)=\sum_{n=0}^{\infty} \lambda_{n}[\epsilon /(k+\epsilon)]^{n},
$$

valid for $\epsilon \geq 0$, and, actually, for $\operatorname{Re}(\epsilon)>-k$.

3. The expansion for the associated characteristic function. Consider the solution of

$$
\begin{gathered}
u^{\prime \prime}+\Lambda(\epsilon)[f(x)+\epsilon g(x)] u=0, \\
u(0)=0, \quad u^{\prime}(0)=1 .
\end{gathered}
$$

The classical iteration procedure for obtaining $u$ shows that $u$ is an analytic function of $\Lambda(\epsilon)$ and $\epsilon$ for $0 \leq x \leq 1$, for all $\epsilon$ and $\Lambda(\epsilon)$. Consequently, $u$ is an analytic function of $\epsilon$ for $R e(\epsilon)>b$, uniformly in $0 \leq x \leq 1$, and thus possesses an expansion

$$
u=\sum_{n=0}^{\infty} u_{n}(x)[\epsilon /(k+\epsilon)]^{n},
$$

valid for $\epsilon \geq 0$.

4. Perturbation procedure. To determine the sequences $\left\{\lambda_{n}\right\}$ and $\left\{u_{n}(x)\right\}$, we employ a standard perturbation technique starting with the equation

$$
\begin{gathered}
u^{\prime \prime}+\lambda[f(x)+k \delta g(x) /(1-\delta)] u=0, \\
u(0)=u(1)=0 .
\end{gathered}
$$

\title{
REFERENCES
}

1. R. Bellman, On perturbation methods involving expansions in terms of a parameter, Quart. Appl. Math. XIII, 195-200 (1955)

2. R. Bellman, Research Problem No. 1, Bull. Am. Math. Soc. 63, No. 1, 59 (1957)

3. T. Fort, On a problem of Richard Bellman, Proc. Am. Math. Soc., to appear

\section{THE NUMERICAL SOLUTION OF AN INFINITE SET OF LINEAR SIMULTANEOUS EQUATIONS*}

By B. NOBLE (The Royal College of Science and Technology, Glasgow, Scotland)

The usual method for numerical solution of

$$
\sum_{s=1}^{\infty} a_{r s} x_{s}=b_{r}, \quad(r=1,2, \cdots),
$$

is to solve the first $n$ equations in $n$ unknowns for some fixed $n$, and to regard the results as approximations to the $x_{s}$. The accuracy of this approximation is invariably doubtful. A slight modification of the Choleski ${ }^{1}$ or Crout $^{2}$ method for solving a set of simultaneous linear equations can be used to provide a systematic procedure for solving the first $m \times m$ equations with $m=1,2,3, \cdots$ in succession. It is then possible to estimate in various ways the error involved in solving only a finite number of equations. The following discussion should be intelligible from either the Crout or the Choleski point of view.

*Received February 14, 1956; revised manuscript received March 7, 1958.

1L. Fox, H. D. Huskey, and J. H. Wilkinson, Quart. J. Mech. and Appl. Math. 1, 149-173 (1948).

${ }^{2}$ P. D. Crout, Trans. Am. Inst. Elec. Engrs. 60, 1235-40 (1941). 
Using matrix notation, the equations $\mathbf{A X}=\mathbf{B}$ are solved by decomposing the matrix $A$ into a product of triangular matrices $\mathbf{A}=\mathbf{L} \mathbf{U}$ where $\mathbf{L}$ is a lower triangular matrix and $\mathrm{U}$ is an upper triangular matrix. The following systems are then solved in succession:

$$
\mathbf{L Y}=\mathbf{B}: \quad \mathbf{U X}=\mathbf{Y} .
$$

We shall adopt the layout used by Crout in which we start with an "original matrix" $\mathbf{D}$ and write down an "auxiliary matrix" $\mathbf{E}$ :

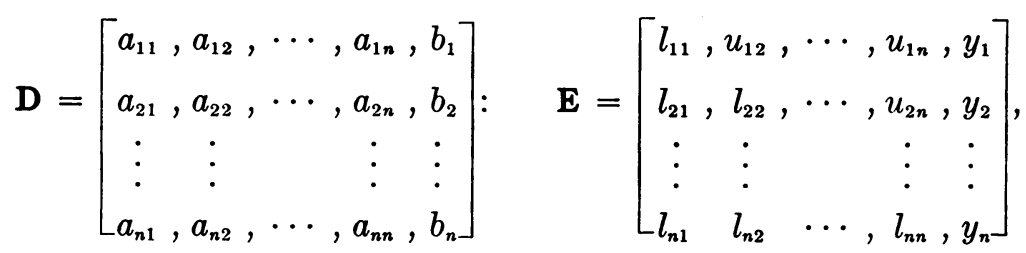

where the $l_{r s}, u_{r_{s}}, y_{s}$, are elements of $\mathbf{L}, \mathbf{U}, \mathbf{Y}$, respectively. For instance $\mathbf{U}$ consists of zeros below the principal diagonal, units in the diagonal and the $u_{r s}$ above the diagonal.

Elements of the auxiliary matrix are determined in the following order:

(i) Elements in the $r$ th column on and below the principal diagonal (i.e. $\left.l_{r, r}, l_{r+1, r}, \cdots, l_{n, r}\right)$.

(ii) Elements in the $r$ th row to the right of the principal diagonal (i.e. $\left.u_{r, r+1}, \cdots, u_{r, n}, y_{r}\right)$.

This sequence is performed for $r=1$, then for $r=2$, and so on, up to $r=n$, by means of the formulae

$$
l_{r s}=a_{r s}-\sum_{m=1}^{s-1} l_{r m} u_{m s}, \quad(r \geq s): \quad u_{r s}=\frac{1}{l_{r r}}\left[a_{r s}-\sum_{m=1}^{r-1} l_{r m} u_{m s}\right], \quad(r<s) .
$$

The proposed method replaces the solution of $\mathrm{UX}=\mathrm{Y}$ by the following procedure $\left(x_{r}^{(m)}\right.$ is the $m$ th approximation to $x_{r}$, etc.).

(a) Take as a first approximation to the solution of the infinite system:

$$
x_{1}^{(1)}=y_{1}: \quad x_{r}^{(1)}=0, \quad(r=2,3, \cdots) .
$$

(b) Obtain a second approximation by solving:

Then

$$
\begin{aligned}
z_{1}^{(2)}+u_{12} z_{2}^{(2)} & =0 \\
z_{2}^{(2)} & =y_{2} .
\end{aligned}
$$

$$
x_{1}^{(2)}=x_{1}^{(1)}+z_{1}^{(2)}: \quad x_{2}^{(2)}=y_{2}: \quad x_{r}^{(2)}=0, \quad(r=3,4, \cdots) .
$$

(c) Obtain a third approximation by solving:

Then

$$
\begin{aligned}
z_{1}^{(3)}+u_{12} z_{2}^{(3)}+u_{13} z_{3}^{(3)} & =0 \\
z_{2}^{(3)}+u_{23} z_{3}^{(3)} & =0 \\
z_{3}^{(3)} & =y_{3} .
\end{aligned}
$$

$$
\begin{array}{ll}
x_{1}^{(3)}=x_{1}^{(2)}+z_{1}^{(3)}: & x_{2}^{(3)}=x_{2}^{(2)}+z_{2}^{(3)}: \\
x_{3}^{(3)}=y_{3}: & x_{r}^{(3)}=0, \quad(r=4,5,6, \cdots) \quad \text { and so on. }
\end{array}
$$


The theory behind the method is obvious if we reflect that at the $m$ th stage we have obtained the exact solution of the first $m$ equations in $m$ unknowns.

As an example consider the following original matrix which is derived from the first four equations of an infinite system ${ }^{3}$ (the elements in the check column are just the sums of the elements in the corresponding rows of the original matrix):

$$
\begin{array}{ccccrc}
x_{1} & x_{2} & x_{3} & x_{4} & \text { Constant } & \text { Check } \\
+5.665,118 & -0.240,000 & +0.059,172 & -0.022,400 & +2.670,644 & 8.132,534 \\
-0.240,000 & +1.270,836 & -0.103,806 & +0.049,941 & -0.049,299 & 0.927,672 \\
+0.059,172 & -0.103,806 & +0.708,321 & -0.051,132 & +0.006,400 & 0.618,955 \\
-0.022,400 & +0.049,941 & -0.051,132 & +0.489,615 & -0.001,666 & 0.464,358 .
\end{array}
$$

The auxiliary matrix is:

$$
\begin{array}{lllllll}
+5.665,118 & -0.042,365 & +0.010,445 & -0.003,954 & +0.471,419 & 1.435,545 \\
-0.240,000 & +1.260,668 & -0.080,353 & +0.038,862 & +0.050,641 & 1.009,150 \\
+0.059,172 & -0.101,299 & +0.699,563 & -0.067,129 & -0.023,393 & 0.909,478 \\
-0.022,400 & +0.048,992 & -0.046,961 & +0.484,470 & +0.010,969 & 1.010,969 .
\end{array}
$$

The procedure described above then gives

$$
\begin{aligned}
& x_{1}^{(1)}=+0.471,419 \text {. } \\
& z_{1}^{(2)}=+0.002,145: \quad x_{2}^{(2)}=+0.050,641 \text {. } \\
& z_{1}^{(3)}=+0.000,165: \quad z_{2}^{(3)}=-0.001,880: \quad x_{3}^{(3)}=-0.023,393 \text {. } \\
& z_{1}^{(4)}=+0.000,020: \quad z_{2}^{(4)}=-0.000,367: \quad z_{3}^{(4)}=+0.000,736: \quad x_{4}^{(4)}=+0.010,969 \text {. }
\end{aligned}
$$

The values for $x_{s}^{(4)}$ agree with those given $i^{3}$ to a unit in the sixth decimal place. In addition, an estimate of accuracy can be obtained by comparing the magnitudes of the corrections for successive values of $m=1,2,3, \cdots$. By inspection of the above results it appears that the approximate $x_{s}$ obtained by solving the $4 \times 4$ set of equations are accurate to roughly $5,4,3,3$ decimal places for $s=1,2,3,4$ respectively.

When the corrections $z_{s}^{(m)}$ do not converge rapidly to zero it will be desirable to give definite upper and lower limits for the unknowns. This may be easy if the corrections alternate in sign. In more difficult cases the following procedure has proved useful. Suppose that we have found the estimates $x_{s}^{(m)}$ of $x_{s}$ by solving the first $m$ equations in $m$ unknowns. Suppose that from these or otherwise it is possible to estimate the $x_{\mathrm{s}}$ for $s=m+1, m+2, \cdots$. Denote these estimates by $X_{i}^{(m)}(s>m)$. Then a second estimate of the $x_{s}$ is given by the quantities $\left(x_{s}^{(m)}+u_{t}^{(m)}\right)$ where the $u_{a}^{(m)}$ satisfy

$$
\sum_{s=1}^{m} a_{r s} u_{s}^{(m)}=-\sum_{s=m+1}^{\infty} a_{r s} X_{s}^{(m)}, \quad(r=1 \text { to } m) .
$$

For sufficiently large $m$ the $u_{s}^{(m)}$ are small corrections and a comparatively crude estimate of the sum on the right hand side of this equation is adequate. If convenient we can

${ }^{3}$ A. Weinstein and D. H. Rock, Quart. Appl. Math. 2, 262-266 (1944). 
replace the $a_{r}$ by an approximation with the correct behavior for large $s$. It will often be possible to replace the summation by integration.

As an example consider the following equations:

$$
\begin{aligned}
& 2.759 x_{1}+0.355 x_{2}+0.302 x_{3}+0.267 x_{4}=1.0000 \\
& 0.214 x_{1}+4.457 x_{2}+0.227 x_{3}+0.214 x_{4}=0.3333 \\
& 0.143 x_{1}+0.178 x_{2}+5.691 x_{3}+0.178 x_{4}=0.2000 \\
& 0.107 x_{1}+0.142 x_{2}+0.151 x_{3}+6.703 x_{4}=0.1429
\end{aligned}
$$

These are the first four equations in four unknowns from an infinite system for which $b_{r}=(2 r-1)^{-1}$ and it is known that for large $r$,

$$
a_{r r} \approx(2 \pi)^{1 / 2}(2 r-1)^{1 / 2}: \quad a_{r s} \approx(2 s-1)^{1 / 2} /\left\{(2 \pi)^{1 / 2}(r+s-1)\right\} .
$$

Also all unknowns are positive so that by inspection

$$
x_{s}<a_{s s}^{-1} b_{s} \approx(2 \pi)^{-1 / 2}(2 s-1)^{-3 / 2} .
$$

We shall use the following estimate in (2):

$$
X_{s}^{(m)} \approx x_{m}^{(m)}(2 m-1)^{3 / 2}(2 s-1)^{-3 / 2} .
$$

This will give an overestimate of the magnitude of the right hand side of (2) since from (4) the $x$, decrease at least as rapidly as $(2 s-1)^{-3 / 2}$ and from the numerical results given below it will appear that the $x_{m}^{(m)}$ are overestimates of the $x_{m}$. We replace the right hand side of (2) by

$$
\begin{aligned}
-x_{m}^{(m)} \frac{(2 m-1)^{3 / 2}}{(2 \pi)^{1 / 2}} \int_{m+1 / 2}^{\infty} \frac{d x}{(2 x-1)(r+x-1)} \\
=-x_{m}^{(m)} \frac{(2 m-1)^{3 / 2}}{(2 \pi)^{1 / 2}(2 r-1)} \log \left(\frac{2 r+2 m-1}{2 m}\right) .
\end{aligned}
$$

The final results are presented in the following table where the upper figures give $x_{s}^{(m)}$, the lower give $x_{s}^{(m)}+u_{0}^{(m)}$. The convergence of the results from above and below as $m$ is increased indicates that upper and lower limits have been established for the unknowns. But the convergence is much slower than in the example given above and it would be difficult to establish accurate estimates of the unknowns from the upper limits alone.

$$
\begin{aligned}
& x_{1}^{(1)}=\begin{array}{l}
0.3624 \\
0.3412
\end{array} \\
& x_{1}^{(2)}=\begin{array}{l}
0.3550 \\
0.3459
\end{array} \quad x_{2}^{(2)}=\begin{array}{l}
0.0577 \\
0.0531
\end{array} \\
& x_{1}^{(3)}=\begin{array}{l}
0.3524 \\
0.3469
\end{array} \quad x_{2}^{(3)}=\begin{array}{l}
0.0566 \\
0.0536
\end{array} \quad x_{3}^{(3)}=\begin{array}{l}
0.0246 \\
0.0225
\end{array} \\
& x_{1}^{(4)}=\begin{array}{l}
0.3512 \\
0.3474
\end{array} \quad x_{2}^{(4)}=\begin{array}{l}
0.0560 \\
0.0539
\end{array} \quad x_{3}^{(4)}=\begin{array}{l}
0.0242 \\
0.0226
\end{array} \quad x_{4}^{(4)}=\begin{array}{l}
0.0140 \\
0.0128 .
\end{array}
\end{aligned}
$$

'D. S. Jones, Proc. Roy. Soc. 217, 153-175 (1953). 
We next consider evaluation of the following infinite series $\left(\mathrm{see}^{4}\right)$ :

$$
S=\sum_{s=1}^{\infty} k_{s} x_{s},
$$

where

$$
k_{1}^{-1}=2.33, \quad k_{2}^{-1}=4.22, \quad k_{3}^{-1}=5.51, \quad k_{4}^{-1}=6.55,
$$

and $k_{o}^{-1} \sim(2 \pi)^{1 / 2}(2 s-1)^{1 / 2}$ for large $s$. If we evaluate

$$
S_{n}=\sum_{s=1}^{n} k_{s} x_{s}^{(n)}
$$

we obtain the following results:

$$
S_{1}=0.155_{5}: \quad S_{2}=0.166_{1}: \quad S_{3}=0.169_{1}: \quad S_{4}=0.170_{5} .
$$

These underestimate $S$. In order to obtain overestimates of $S$ we evaluate, using the previous approximation (5),

$$
\begin{aligned}
S_{n}^{\prime} & =\sum_{s=1}^{n} k_{s} x_{s}^{(n)}+k_{n} x_{n}^{(n)}(2 n-1)^{2} \sum_{s=n+1}^{\infty} \frac{1}{(2 s-1)^{2}} \\
& \approx \sum_{s=1}^{n} k_{s} x_{s}^{(n)}+k_{n} x_{n}^{(n)}(2 n-1)^{2}(4 n)^{-1},
\end{aligned}
$$

where we have replaced summation by integration as before. This gives

$$
S_{1}^{\prime}=0.194_{4}: \quad S_{2}^{\prime}=0.181_{5}: \quad S_{3}^{\prime}=0.178_{5}: \quad S_{4}^{\prime}=0.176_{9} .
$$

The mean values $\frac{1}{2}\left(S_{n}+S_{n}^{\prime}\right)$ are remarkably constant. We can say from these results that $0.171<S<0.177$ with a probable value of $S=0.174$.

\section{ON SIMPLE SUBHARMONICS*}

By C. S. HSU** (University of Toledo, Toledo, Ohio)

Introduction. In a recent paper [1], $\uparrow$ Rosenberg clarified considerably the curious subharmonic phenomena of non-linear oscillations by introducing the concepts of strong subharmonic solutions and of the simple subharmonics. He considered a system with a single degree of freedom, whose mechanical model might be a mass under the action of an elastic force, linear or non-linear, and of a simple harmonic forcing function of frequencey $\omega$. The equation of motion of the system can be put in the form

$$
\frac{d^{2} x}{d t^{2}}+f(x)=P_{0} \cos \omega t,
$$

where $P_{0}$ is proportional to the amplitude of the external forcing function.

*Received June 16, 1958.

**Now affiliated with University of California, Berkeley.

$\uparrow$ Numbers in square brackets refer to the bibliography at the end of the paper. 